\title{
Bilateral Inguinal Lymphoceles Following Robotic Radical Prostatectomy
}

\author{
Sameer Chopra, MD, Patrick Ramos, MD, Andre Luis de Castro Abreu, MD, Scott Leslie, MD, \\ Monish Aron, MD \\ USC Institute of Urology, Center for Advanced Robotic Surgery, Keck School of Medicine, University of Southern \\ California, Los Angeles, CA, USA (all authors).
}

\begin{abstract}
Introduction: Pelvic lymphoceles are a known complication of pelvic lymph node dissection after robotic-assisted radical prostatectomy (RARP). However, large symptomatic inguinal lymphoceles after RARP have hitherto not been reported.

Case Description: We present the case of a 71-year-old morbidly obese man who developed large, symptomatic, bilateral inguinal lymphoceles after RARP and pelvic lymph node dissection. The surgery itself was uneventful, as was the hospital stay. The patient returned 3 weeks postoperatively with bilateral inguinal pain and swelling, which was confirmed on imaging to be bilateral inguinal lymphoceles. These were initially treated with bilateral percutaneous pigtail catheter drainage, but this treatment was complicated by repeated tube blockages, fever, and conversion of the lymphoceles into multiloculated abscesses. Definitive treatment consisted of open left lymphocele excision first. After excision of the left inguinal lymphocele, the right lymphocele became infected and formed a large inflammatory phlegmon, necessitating open right inguinal lymphocele excision with right orchiectomy. Culture demonstrated gram-negative Prevotella bivia.

Discussion: This case was unique because the patient presented with bilateral, large, symptomatic, recurrent inguinal lymphoceles, as opposed to the more common pelvic lymphoceles. To our knowledge, this is the first reported case of bilateral, symptomatic inguinal lymphoceles after RARP with pelvic lymph node dissection.
\end{abstract}

Key Words: Lymphocele, Radical prostatectomy, Robotics, Complications.

Citation Chopra S, Ramos P, de Castro Abreu AL, Leslie S, Aron M. Bilateral inguinal lymphoceles following robotic radical prostatectomy. CRSLS e2014.00357. DOI 10.4293/CRSLS.2014.00357.

Copyright (C) 2014 SLS This is an open-access article distributed under the terms of the Creative Commons Attribution-Noncommercial-ShareAlike 3.0 Unported license, which permits unrestricted noncommercial use, distribution, and reproduction in any medium, provided the original author and source are credited.

Address correspondence to: Monish Aron, MD, USC Institute of Urology, Center for Advanced Robotic Surgery, Keck School of Medicine, University of Southern California, 1441 Eastlake Ave, Suite 7416, Los Angeles, CA 90089; Telephone: 323-865-3700, Fax: 323-865-0120, E-mail: monisharon@hotmail.com.

\section{INTRODUCTION}

Lymphoceles within the pelvis are a known complication of pelvic lymph node dissection (PLND) performed concomitantly with robotic-assisted radical prostatectomy (RARP). ${ }^{1}$ However, few lymphoceles are symptomatic or require intervention, and the vast majority of these are confined to the pelvis. ${ }^{2}$ Lymphoceles that do become symptomatic are typically managed successfully by simple interventions such as percutaneous drainage and rarely require open surgery. ${ }^{3}$ Herein, we describe the presentation, clinical course, and treatment of symptomatic, large, bilateral inguinal lymphoceles after RARP and PLND, re- sulting in a complicated and challenging postoperative course.

\section{CASE DESCRIPTION}

A 71-year-old morbidly obese (body mass index, $39 \mathrm{~kg} /$ $\mathrm{m}^{2}$ ) Caucasian man was found to have clinical stage T1c, Gleason $7(3+4)$ adenocarcinoma of the prostate after transrectal ultrasound-guided biopsy for a prostate-specific antigen level of $6.5 \mathrm{ng} / \mathrm{mL}$. The patient had a surgical history of open appendectomy, bilateral hip replacements, and a right inguinal hernia repair. Although the patient was morbidly obese with a narrow pelvis, he 

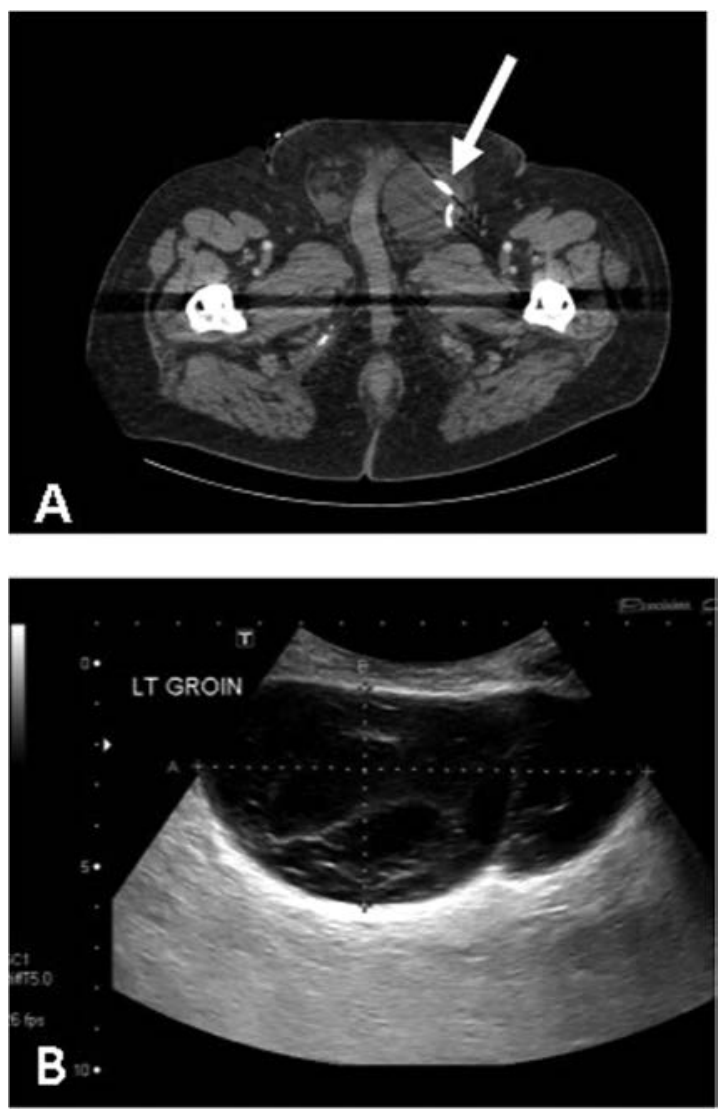

Figure 1. (A) Computed tomographic scan demonstrating persistent left inguinal lymphocele despite a pigtail catheter. (B) Ultrasound demonstrating the septated nature of the lymphocele collection.

underwent an uncomplicated transperitoneal RARP and bilateral PLND. Final pathology was Gleason $7(3+4)$ adenocarcinoma confined to the prostate (pT2c), with 56 lymph nodes (left, 27; right, 29) negative for disease. The patient was discharged from the hospital on postoperative day 2 after the removal of his 19-F pelvic drain. Drain output was $67 \mathrm{~mL}$ in the 24 hours before its removal.

Over the course of the next 2 months, the patient required multiple readmissions for bilateral inguinal swelling and pain. On computed tomographic (CT) imaging, 3 weeks postoperatively, he was found to have bilateral inguinal lymphoceles. Initial conservative management with analgesia and scrotal support was unsuccessful as the groin lymphoceles increased in size and became more symptomatic. Percutaneous aspiration was then performed bilaterally, with pigtail drains placed using ultrasound guidance. A total of $300 \mathrm{~mL}$ of fluid was aspirated from the right groin lymphocele, and $600 \mathrm{~mL}$ of fluid was aspirated from the left. The aspirate was found to be sterile, and analysis was consistent with lymphatic fluid.

After drainage, the patient's right-sided symptoms completely resolved. However, the patient's left inguinal pain and swelling persisted, and there were several instances of pigtail catheter blockages requiring tube changes. A repeat CT scan demonstrated an actual increase in the size of the left lymphocele, and therefore the pigtail catheter was exchanged to a larger size $(14 \mathrm{~F})$. This proved to be of little benefit, and the patient had worsening pain and swelling in the left groin. A repeat CT scan demonstrated a large, complex fluid collection within the left groin (Figure 1A), and ultrasound demonstrated the multiloculated nature of this fluid collection (Figure 1B). Because the pigtail was now totally ineffective, an open left inguinal exploration was performed. The large lymphocele sac was identified within the inguinal canal and separated from the cord structures and testicle (Figure 2A). The sac was markedly inflamed, and on opening the lymphocele, the sac was noted to have a very thick wall with multiple internal septations consistent with the imaging findings (Figure 2B). The sac was excised completely, and pathology was consistent with a lymphocele sac.
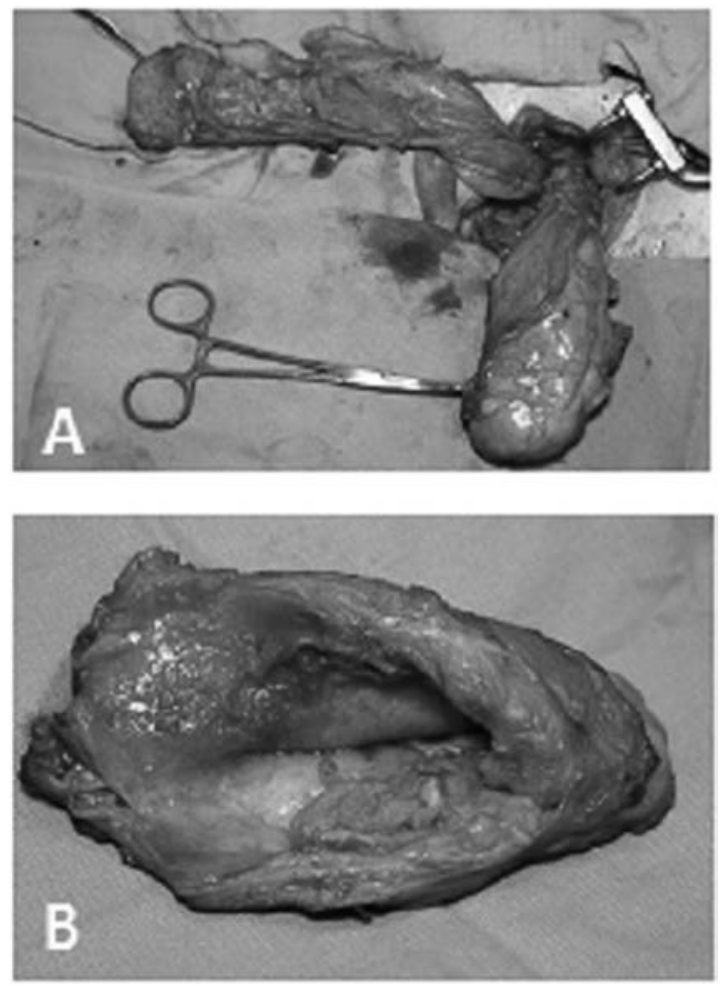

Figure 2. Left inguinal exploration. (A) Inguinal lymphocele sac separated from spermatic cord and testicle. (B) Excised lymphocele sac demonstrating thickened wall. 
Postoperatively, the left-sided pain resolved, but during the patient's hospital course, his right groin pain recurred. This was associated with a fever $\left(39.5^{\circ} \mathrm{C}\right)$, an elevated white blood cell count $\left(14.3 \times 10^{3}\right.$ cells $\left./ \mu \mathrm{L}\right)$, and erythema and tenderness in the right inguinal region (Figure 3). Radiologic imaging showed that the right lymphocele had increased in size, with associated fat stranding consistent with an abscess (Figure 4).

The patient was taken to the operating room for right inguinal exploration. Intraoperatively, we found an inflammatory phlegmon completely encasing the spermatic cord within the inguinal canal. Separation of the phlegmon from the underlying spermatic cord structures was not possible, and intraoperative testicular ultrasound confirmed minimal arterial flow. The right testicle and spermatic cord were therefore excised en bloc with the phlegmon (Figures 5A and 5B). Pathologic results were consistent with an abscess associated with thrombosed testicular vessels. Cultures from the abscess cavity identified Prevotella bivia (gram-negative bacteria). Finally, the patient had an uneventful recovery. His last follow-up visit was 9 months postoperatively. All symptoms related to the inguinal lymphoceles had resolved. He was fully continent, and his prostate-specific antigen was undetectable $(<0.03 \mathrm{ng} / \mathrm{mL})$ at this time.

\section{DISCUSSION}

Lymphoceles are a recognized complication after PLND and RARP. However, the incidence of lymphocele formation is underestimated, because imaging is rarely implemented for patients undergoing RARP. In studies that routinely performed either ultrasound or CT scans postoperatively to identify the true incidence of lymphoceles, rates varied between $13 \%$ and $61 \% .4,5$ Orvieto et $\mathrm{al}^{2}$ as-

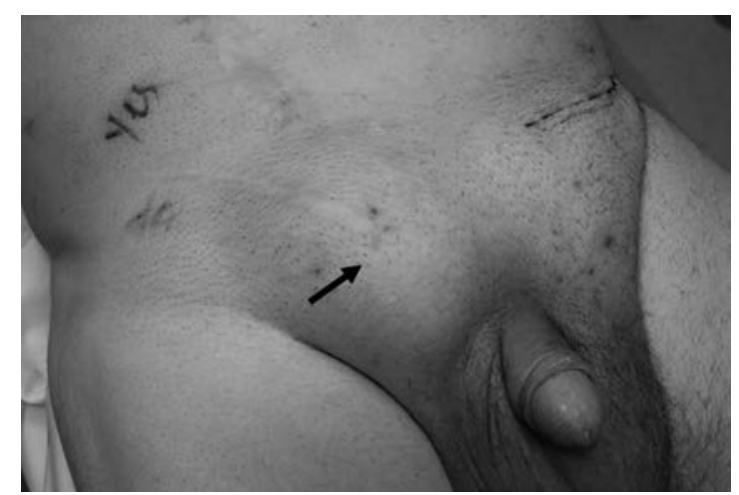

Figure 3. Swelling (arrow) of right inguinal lymphocele associated with erythema of overlying skin.

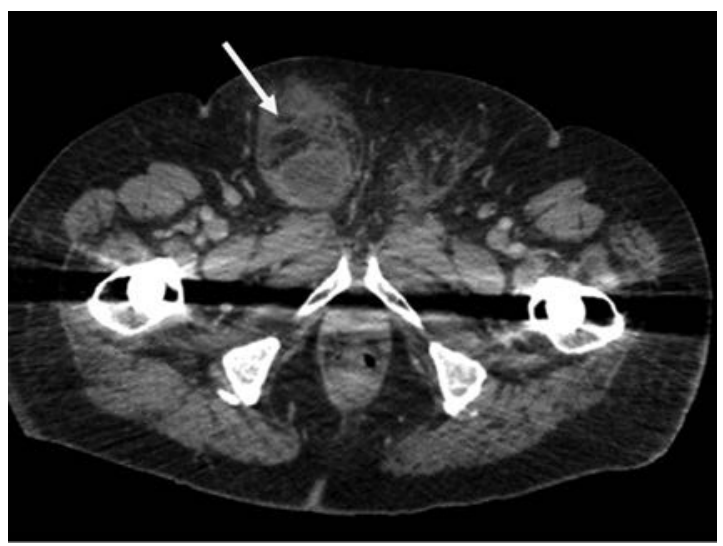

Figure 4. Computed tomographic scan demonstrating increased size of right inguinal lymphocele associated with marked stranding. Postoperative changes are noted on the left side.
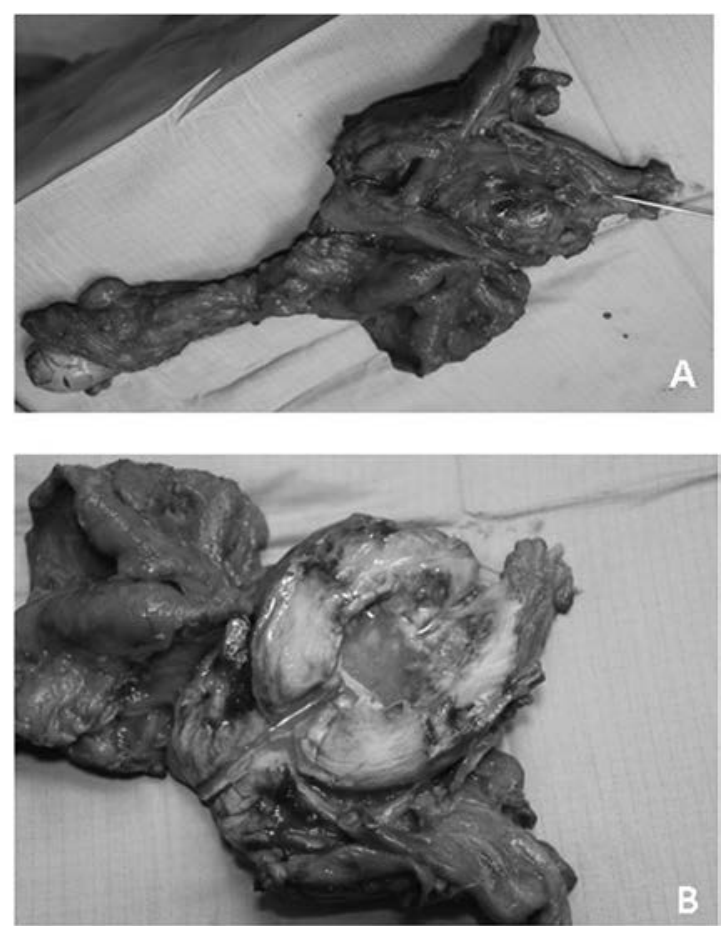

Figure 5. (A) En bloc excision of right inguinal phlegmon with the right testicle and spermatic cord. (B) Inflammatory phlegmon bivalved demonstrating abscess cavity and grossly thickened wall.

sessed the incidence of lymphoceles after RARP and pelvic lymphadenectomy. In that study, CT scans were performed in 76 patients, 6 to 12 weeks after RARP or PLND, and 39 patients (51\%) were found to have radiologic evidence of lymphoceles. However, only 6 patients reported symptoms related to the lymphoceles, indicating 
that in that study, the rate of clinically significant lymphoceles was $7.8 \%$. This is consistent with other series that have reported rates of clinically significant lymphoceles between $1 \%$ and $6 \%, 1,6$

The rate of perioperative complications after PLND has been reported to be $4 \%$ to $20 \%$. $^{7-9}$ In addition to lymphocele formation, lymphadenectomy has other known complications, some of which can be life threatening. Some of the complications described include vascular injuries, iliac vein thrombosis, nerve injuries, neuropathy, and the development of lymphedema. ${ }^{7-11}$ Furthermore, although most lymphoceles appear to be subclinical and require no intervention, some can lead to symptoms and secondary complications such as abdominal pain, leg pain, lower limb edema, constipation, urinary frequency, deep vein thrombosis, and infection or sepsis. ${ }^{7,8}$ Because of the severity of these potential complications, all efforts must be made to minimize the occurrence of lymphoceles.

Risk factors for lymphocele formation include age, number of lymph nodes retrieved, and the use of low-molecular weight heparin. ${ }^{12,13}$ Capitanio et al ${ }^{12}$ studied 501 patients after open radical prostatectomy and found age and lymph node count to be the most significant predictors. They also found that lymphadenectomy along the external iliac artery conferred a greater risk for lymphocele formation compared with dissection within the obturator region. Gotto et $\mathrm{al}^{13}$ retrospectively reviewed the charts of $>4,000$ radical prostatectomy patients, both open and laparoscopic, and found a correlation between the number of lymph nodes removed and the risk for lymphocele development. Additionally, they identified the use of lowmolecular weight heparin as a risk factor for lymphocele formation.

In our case, the recognized risk factors for lymphocele formation were present. The patient was 71 years of age, prophylactic low-molecular weight heparin was given, and a total of 56 lymph nodes were removed during PLND. However, what was unusual about this patient's presentation was the presence of large symptomatic bilateral inguinal lymphoceles, as opposed to the more common pelvic lymphoceles. Although decidedly rare, as this report demonstrates, inguinal lymphoceles can occur after PLND.

Conventional management of symptomatic lymphoceles as described in the literature includes percutaneous aspiration with or without the placement of a drainage catheter $^{3}$; instillation of sclerosing agents within the lymphocele ${ }^{14}$; and, for lymphoceles recalcitrant to percutaneous drainage, laparoscopic marsupialization of the lympho- cele sac into the peritoneal cavity. ${ }^{15}$ The use of percutaneous drainage as initial treatment must be judicious, as studies have demonstrated high lymphocele recurrence rates after percutaneous drainage. 6,16

Many authors suggest that the best approach for treating symptomatic, sterile lymphoceles is drainage using laparoscopic techniques. ${ }^{15,17-18}$ In a case series of patients with pelvic lymphoceles after RARP, Raheem et $\mathrm{al}^{17}$ found that 1 patient who underwent laparoscopic marsupialization of bilateral pelvic lymphoceles had no complications and was discharged earlier than 3 patients who received percutaneous drainage. Our patient was offered early surgical exploration but, after discussion of all options, elected to proceed with percutaneous pigtail drainage in the first instance.

The pathophysiology of the development of our patient's inguinal lymphoceles is uncertain. Our conjecture is that small peritoneal defects at the deep inguinal ring allowed the pelvic lymphatic fluid to gravitate down to the inguinal canal postoperatively. Bilateral groin pain and swelling was the clinical manifestation of these postoperative lymphoceles. Percutaneous drainage and pigtail insertion led to the resolution of right-sided symptoms initially, but his left inguinal lymphocele increased in size. Drainage was unsuccessful, likely because of the subsequent multiloculated nature of the lymphocele. Furthermore, because these were symptomatic inguinal lymphoceles, laparoscopic marsupialization was not an option.

The unique nature and location of this patient's persistent lymphocele necessitated excision of the lymphocele sac. Immediate resolution of the patient's left-sided symptoms were seen, but the surgery itself may have introduced infection to the contralateral inguinal lymphocele, which had largely become asymptomatic after initial aspiration and pigtail drainage.

The subsequent phlegmon that developed on the right side unfortunately necessitated en bloc excision of the testicle, cord, and inflammatory mass. However, complete resolution of all symptoms was seen after this surgery.

\section{CONCLUSIONS}

To our knowledge, this is the first report in the literature of bilateral, symptomatic inguinal lymphoceles complicating RARP and PLND. Intervention with aspiration and placement of percutaneous drains was performed initially and given an appropriate trial. However, as these initial measures failed, we found that lymphocele 
excision was the definitive surgical option that led to eventual resolution of our patient's complicated clinical course.

\section{References:}

1. Zorn KC, Katz MH, Bernstein A, et al. Pelvic lymphadenectomy during robot-assisted radical prostatectomy: assessing nodal yield, perioperative outcomes, and complications. Urology. 2009;74(2):296-302.

2. Orvieto MA, Coelho RF, Chauhan S, Palmer KJ, Rocco B, Patel VR. Incidence of lymphoceles after robot-assisted pelvic lymph node dissection. BJU Int. 2011;108(7):1185-1190.

3. Kim JK, Jeong YY, Kim YH, Kim YC, Kang HK, Choi HS. Postoperative pelvic lymphocele: treatment with simple percutaneous catheter drainage. Radiology. 1999;212(2):390-394.

4. Gust K. Clinical significance of postoperative lymphoceles following pelvic lymph node dissection in prostate cancer disease. Int J Urol. 2009;1(1):94-102.

5. Solberg A, Angelsen A, Bergan U, Haugen OA, Viset T, Klepp O. Frequency of lymphoceles after open and laparoscopic pelvic lymph node dissection in patients with prostate cancer. Scand J Urol. 2003;37(3):218-221.

6. Yee DS, Katz DJ, Godoy G, et al. Extended pelvic lymph node dissection in robotic-assisted radical prostatectomy: surgical technique and initial experience. Urology. 2010;75(5):11991204.

7. Heidenreich A, Varga Z, von Knobloch R. Extended pelvic lymphadenectomy in patients undergoing radical prostatectomy: high incidence of lymph node metastasis. J Urol. 2002;167(4): 1681-1686.

8. Naselli A, Andreatta R, Introini C, Fontana V, Puppo P. Predictors of symptomatic lymphocele after lymph node excision and radical prostatectomy. Urology. 2010;75(3):630635.
9. Briganti A, Chun FK, Salonia et al. Complications and other surgical outcomes associated with extended pelvic lymphadenectomy in men with localized prostate cancer. Eur Urol. 2006; 50(5):1006-1013.

10. Freid RM, Siegel D, Smith AD, Weiss GH. Lymphoceles after laparoscopic pelvic lymph node dissection. Urology. 1998;51(5 suppl 1):131-134.

11. Capitanio U, Suardi N, Montorsi F, Briganti A. Editorial comment to Risk factors for pelvic lymphoceles post-radical prostatectomy. Int J Urol. 2011;18(9):644-645.

12. Gotto GT, Yunis LH, Guillonneau B, et al. Predictors of symptomatic lymphocele after radical prostatectomy and bilateral pelvic lymph node dissection. Int J Urol. 2011;18(4):291296.

13. Gilliland JD, Spies JB, Brown SB, Yrizarry JM, Greenwood LH. Lymphoceles: percutaneous treatment with povidone-iodine sclerosis. Radiology. 1989;171(1):227-229.

14. Fallick ML, Long JP. Laparoscopic marsupialization of lymphocele after laparoscopic lymph node dissection. J Endourol. 1996;10(6):533-534.

15. Feicke A, Baumgartner M, Talimi S, et al. Robotic-assisted laparoscopic extended pelvic lymph node dissection for prostate cancer: surgical technique and experience with the first 99 cases. Eur Urol. 2009;55(4):876-883.

16. Raheem OA, Bazzi WM, Parsons JK, Kane CJ. Management of pelvic lymphoceles following robot-assisted laparoscopic radical prostatectomy. Urol Ann. 2012;4(2):111-114.

17. Thurlow JP, Gelpi J, Schwaitzberg SD, Rohrer RJ. Laparoscopic fenestration and internal drainage of lymphoceles after renal transplantation. Surg Laparosc Endosc. 1996;6(4):290-295.

18. Gruessner RW, Fasola C, Benedetti E et al. Laparoscopic drainage of lymphoceles after kidney transplantation. Indications and limitations. Surgery. 1995;117(3):288-295. 